\title{
Projetos políticos pedagógicos e sua interface com as diretrizes curriculares nacionais de enfermagem
}

\author{
Political pedagogical projects and their interface with the national nursing curriculum guidelines
}

Proyectos políticos pedagógicos y su interrelación con las directivas curriculares nacionales de enfermería

Ana Maria Maia Rodrigues', Consuelo Helena Aires de Freitas², Maria Salete Bessa Jorge, Maria das Graças da Silva Guerreiro ${ }^{4}$, Danielle Christine Moura dos Santos ${ }^{5}$

\footnotetext{
${ }^{1}$ Enfermeira, Discente do Programa de Pós-Graduação Cuidados Clínicos em Enfermagem e Saúde da Universidade Estadual do Ceará (PPCCLIS/UECE), nível Mestrado. Fortaleza, CE, Brasil. E-mail: ana maria cartaxo@hotmail.com.

2 Enfermeira, Doutora em Enfermagem. Professora Adjunta do Departamento de Enfermagem da UECE. Fortaleza, CE, Brasil. E-mail: consueloaires@yahoo.com.br.

3 Enfermeira, Doutora em Enfermagem. Professora Titular do Departamento de Enfermagem da UECE. Fortaleza, CE, Brasil. E-mail: maria.salete.jorge@gmail.com.

${ }^{4}$ Enfermeira, Mestre em Cuidados Clínicos em Enfermagem e Saúde. Fortaleza, CE, Brasil. E-mail: mgsguerreiro@yahoo.com.br.

${ }^{5}$ Enfermeira, Mestre em Cuidados Clínicos em Enfermagem e Saúde. Discente do PPCCLIS/UECE, nível Doutorado. Fortaleza, CE, Brasil. E-mail: daniupe@yahoo.com.br.
}

\section{RESUMO}

O presente trabalho trata-se de pesquisa documental sobre as diretrizes curriculares para a graduação em enfermagem (DCN/ENF) no Brasil - aprovadas em 2001 pela Resolução nº 03/2001-CNE - e os Projetos Políticos Pedagógicos (PPP) dos cursos de Enfermagem de três Universidades de Fortaleza-CE. O estudo buscou analisar os PPPs e Matrizes Curriculares dos cursos de graduação de Enfermagem e sua correlação com as DCN/ENF. Os resultados demonstraram que as instituições vislumbram sua aproximação com o documento em questão, estruturando o conteúdo dos seus PPPs conforme o preconizado. No entanto, observam-se sinais do modelo de ensino cartesiano e com foco mais evidenciado nas disciplinas de cunho biológico em detrimento daquelas que contemplam as ciências humanas. Desta forma, percebeu-se que a consolidação do SUS, no que se refere à formação de recursos humanos na cidade de Fortaleza, ainda passa por processo em construção. Logo, conclui-se que o PPP deve ser constantemente avaliado para reajustes necessários.

Descritores: Educação em Enfermagem; Formação de Recursos Humanos; Enfermagem; Currículo.

\section{ABSTRACT}

This is a documental study regarding the national curriculum guidelines for nursing undergraduate courses (DCN/ENF) in Brazil approved in 2001 by Resolution 03/2001-CNE - and the Political Pedagogical Projects (PPP) of the nursing courses of three universities from Fortaleza, Ceará. The study aimed to analyze the PPPs and the Original Curriculum of the nursing undergraduate courses and their correlation with the DCN/ENF. Results show that the institutions expect to comply with the referred document, and, therefore, structure the content of their PPPs as recommended. However, it is observed there are signs of the Cartesian education model and a more evident focus on biological disciplines over human sciences. Therefore, it is suggested that the consolidation of the national health system (SUS), in terms of the development of human resources in Fortaleza, is still under construction. Hence, it is concluded that the PPP must be constantly evaluated for the necessary adjustments.

Descriptors: Education, Nursing; Human Resources Formation; Nursing; Curriculum.

\section{RESUMEN}

Investigación documental acerca de las directivas curriculares de graduación en enfermería (DCN/ENF) en Brasil, aprobadas en 2001 mediante Resolución 03/2001-CNE y de los Proyectos Políticos Pedagógicos (PPP) de los cursos de Enfermería de tres universidades de Fortaleza-CE. Se buscó analizar los PPP y Matrices Curriculares de los cursos de Enfermería y su correlación con las DCN/ENF. Los resultados demostraron que las instituciones apuntan a aproximarse al documento en cuestión, estructurando el contenido de sus PPP conforme lo recomendado. Mientras tanto, se observan signos del modelo de enseñanza cartesiana con foco más evidente en las disciplinas de cuño biológico, en detrimento de aquellas que contemplan las ciencias humanas. De tal forma, se percibió que la consolidación del SUS en lo referente a la formación de recursos humanos en Fortaleza-CE, aún se trata de un proceso en construcción, dado que las PPP deben ser constantemente evaluadas para los ajustes necesarios.

Descriptores: Educación en Enfermería; Formación de Recursos Humanos; Enfermería; Curriculum. 


\section{INTRODUÇÃO}

As mudanças ocorridas no campo da saúde, principalmente no contexto da reforma sanitária e implantação do Sistema Único de Saúde (SUS) na década de 80 , suscitaram reflexões e questionamentos sobre a formação do enfermeiro, como as competências do profissional e os princípios que demarcam a sua formação. Neste sentido, debates e discussões acerca do perfil do enfermeiro foram marcados, principalmente, pelo momento histórico e político que o País vivenciou no período de redemocratização.

Os cursos de graduação em Enfermagem se encontravam, em sua maioria, até a implantação do SUS, reproduzindo uma formação fragmentada e focada na organização curricular disciplinar, centrada em especialidades com valorização do saber técnico, tendo como cenário predominante de prática o hospital, enfatizando práticas em saúde procedimento-centradas, não situando a co-responsabilidade usuário, trabalhador, gestor do SUS e sociedade, para a mudança do novo modelo de saúde que surgia ${ }^{(1-2)}$.

A partir da constituição do SUS e de suas atribuições como ordenador dos recursos humanos na área de saúde( ${ }^{(3)}$, percebeu-se a necessidade de legitimar, na prática, uma profunda reestruturação nas matrizes curriculares dos cursos de graduação em saúde, a serem realizadas por meio de sucessivas e permanentes inovações, pautadas no princípio da integralidade, articulando concepções, práticas de saúde e organizações das ações em busca da reconstrução do trabalho e dos processos de gestão em saúde.

A partir da Lei de Diretrizes e Bases (LDB) de 1996, concretizou-se, em 7/8/2001, o Parecer 1133 do $\mathrm{CNE} / \mathrm{CES}^{(4)}$, que veio reforçar a necessidade da articulação entre Educação Superior e Saúde, objetivando a formação geral e específica dos egressos/ profissionais, com ênfase na promoção, prevenção, recuperação e reabilitação da saúde. Após esse Parecer, foi aprovada a Resolução CNE/CES No 03 de 7/11/2001, que definiu as Diretrizes Curriculares Nacionais para o Curso de Graduação em Enfermagem (DCN/ENF) ${ }^{(5)}$. Iniciou-se um movimento de reformas curriculares que se sucederam na última década.

Neste sentido, o Projeto Político Pedagógico (PPP) coloca-se como forma de apontar os objetivos de um curso e nortear estratégias de coordenação das ações dos diversos sujeitos envolvidos no processo educativo, um instrumento de integração(6).

Para o presente estudo, adotamos a concepção de que o PPP deverá buscar a formação integral e adequada do estudante através de uma articulação entre o ensino, a pesquisa e a extensão/assistência, orientando o Currículo do Curso para a inclusão de competências, habilidades e conteúdos, que considerem as demandas e expectativas do setor saúde na região(7). Além dessa, concebemos que o Currículo deverá permear ações entre processos de seleção, distribuição, organização e ensino dos conteúdos curriculares consonante à estrutura de poder existente no contexto social, histórico e cultural do local e da comunidade na qual a instituição está inserida(8).

Resultantes do processo de discussão envolvendo representantes do governo, docentes, estudantes, diretores de cursos e escolas, entre outros, as DCN/ENF destinam-se a orientar a formação desses profissionais, tendo como base conhecimentos gerais e ênfase no compromisso social e na mudança do modelo de saúde. Expressam os conceitos originários dos movimentos por mudanças na educação em enfermagem, explicitando a necessidade do compromisso com princípios da Reforma Sanitária Brasileira e SUS ${ }^{(9-10)}$.

As instituições formadoras vêm sendo impulsionadas para desenvolverem modificações no processo de formação e na maneira como se relacionam com a sociedade. A necessidade de mudança decorre de elementos, tais como as novas modalidades de organização do mundo do trabalho em saúde e as exigências em relação ao perfil dos novos profissionais, os desafios da interdisciplinaridade e da transdisciplinaridade na produção de conhecimento ou a necessidade das Instituições de Ensino Superior (IES) reconstruírem seu papel social, considerando a multiplicidade de lugares produtores do conhecimento no mundo atual(11).

Deste modo, entende-se que o cenário das políticas públicas no campo da saúde não pode ser discutido sem o devido destaque para a questão da formação dos seus trabalhadores, representando assim, um tema instigante pelos questionamentos e conflitos que suscita e pelas transformações que requer para a consolidação do SUS.

No momento em que se completam 10 anos de aprovação das DCN/ENF, as modificações ainda são lentas no que diz respeito à formação em saúde no município de 
Fortaleza, Ceará. Mediante as desigualdades regionais brasileiras $^{(12)}$, compreende-se que a presente investigação virá contribuir com as discussões em torno da reformulação dos PPPs das instituições em estudo. Sabe-se que se constitui de grande desafio o processo de formação integral na saúde por requerer a incorporação de princípios de dimensão ética, filosófica, técnica, social, política, econômica e cultural, mediante uma lógica social e democrática que envolve pensamentos e valores de pessoas que se situam como agentes transformadores ou resistentes à democratização da sociedade.

A clínica ampliada propõe o compromisso dos profissionais da saúde com os seus usuários, estendendo sua responsabilidade e ética no sentido de evitar exclusões e dar resolutividade às suas demandas. Os profissionais devem desenvolver a capacidade de não somente ajudar as pessoas a prevenir e tratar doenças, mas, sobretudo de trabalhar as potencialidades do usuário para continuar a produzir e viver o seu dia a dia. Os elementos da clinica ampliada, adicionados no pensar, podem criar e recriar o fazer da enfermagem, já estabelecido até mesmo com o processo de enfermagem, contribuindo para um cuidar mais efetivo, humano e integral ao usuário, família e coletividade ${ }^{(13)}$.

Este pensar coletivo deve ser iniciado na estrutura curricular dos enfermeiros, com PPPs fundamentados na integralidade consonante ao SUS centrado no usuário e na família, com a ampliação de possibilidades de intervenções do enfermeiro junto à equipe de saúde. Pois, não há um modelo fechado e único quando o ensino aprendizagem acontece para um fazer sem diferenças e com complementaridades ${ }^{(14)}$.

Portanto, esse estudo buscou analisar os Projetos Políticos Pedagógicos e Matrizes Curriculares dos cursos de graduação de Enfermagem de duas Universidades Públicas de Ensino Superior e de uma Universidade Privada da cidade de Fortaleza-CE, estabelecendo relações com as Diretrizes Curriculares Nacionais.

\section{METODOLOGIA}

Trata-se de um estudo documental, no qual se recorre às fontes primárias dos PPPs. Foram analisados Projetos Políticos Pedagógicos (PPPs) e Matrizes Curriculares de dois cursos de graduação em enfermagem de instituições de ensino público e um de instituição privada de Fortaleza-CE, no período de março a outubro de 2011. Contou com a anuência das três universidades após solicitação formal junto aos departamentos responsáveis. O estudo integra o projeto "Processo de formação em saúde como estratégia de melhoria do cuidado interdisciplinar e integral do SUS" aprovado pelo Comitê de Ética em Pesquisa da Universidade Estadual do Ceará processo n 14061052-2 e financiado pelo CNPq.

No intuito de preservar o anonimato dos cursos de graduação em enfermagem no respeito aos aspectos éticos da pesquisa, os Projetos Políticos Pedagógicos foram denominados por PPP1, PPP2 e PPP3.

O percurso metodológico consistiu na obtenção do material junto às Instituições de Ensino Superior (IES), iniciando-se a leitura analítica, tomando por base as DCN do curso de graduação em Enfermagem. Procedeu-se a organização das informações, possibilitando reuni-las em núcleos de sentido: o perfil do egresso, competências e habilidades gerais e competências e habilidades específicas.

Procedeu-se a análise das matrizes curriculares das instituições, buscando as ementas das disciplinas/módulos no sentido de evidenciar aspectos relacionados aos conteúdos curriculares, cargas horárias e distribuição temporal no curso. As disciplinas foram categorizadas a partir da adaptação das categorias utilizadas em outro estudo(15). Assim, obtiveram-se conteúdos: Biológicos e individuais; Saúde coletiva e preventiva; Prevenção e atenção individual/biológica; e Ciências sociais e humanas.

Foi realizada uma leitura do conteúdo manifesto, fichamentos para classificar os núcleos de sentido por meio de palavras-chave, seleção de trechos importantes acerca das relações entre os conteúdos presentes no texto dos PPPs, os objetivos operacionais e filosóficos do curso, bem como as questões relacionadas ao perfil do profissional dos egressos dos cursos.

Para a análise utilizou-se técnicas usuais de análise de conteúdo com o intuito de decifrar, em cada texto, o núcleo emergente que atendesse ao propósito do estudo. Essa etapa consistiu no processo de codificação, interpretação e de inferências sobre as informações contidas nos documentos, desvelando seu conteúdo manifesto e latente ${ }^{(16)}$, resultando em duas categorias temáticas: as singularidades dos Projetos Políticos Pedagógicos e as matrizes curriculares e sua interface com os objetivos educacionais. 
O tratamento dos dados deu-se a partir da busca dos aspectos objetivos e subjetivos contidos nos PPPs, em que foi possível desvelar questões relacionadas ao processo de formação como um todo, desmistificando se tal instituição já traz em seus escritos e em suas propostas de implementação, os caminhos requeridos para o alcance da assistência integral preconizada pelo SUS desde sua concepção.

\section{RESULTADOS E DISCUSSÃO}

\section{As singularidades dos Projetos Político Pedagógicos}

O Projeto Político Pedagógico de uma instituição deve ser construído e vivenciado por todos os envolvidos com o processo educativo. Busca uma direção, por meio de ação intencional com um compromisso definido coletivamente. Assim, todo projeto pedagógico é, também, um projeto político por estar intimamente articulado ao compromisso sociopolítico com a formação do cidadão para um tipo de sociedade ${ }^{(7)}$.

Os PPPs do estudo abordam em sua apresentação o conceito acerca do termo Projeto Político Pedagógico, o que vai ao encontro do que vem sendo discutido por educadores acerca do tema. Destaca-se, a seguir, uma das concepções apresentadas: [...] instrumento de balizamento para o fazer universitário, concebido coletivamente no âmbito da instituição, orientado por esta, como um todo, e para cada um dos seus cursos em particular. Deve ensejar a construção da intencionalidade para o desempenho do papel social da Instituição, centrando-se no ensino, mas vinculando-se estreitamente aos processos de pesquisa $e$ extensão. Com base na análise crítica do momento vivido, deve-se configurar a visão pretendida, efetivando as ações, refletindo sobre elas, avaliando-as e incorporando novos desafios [....] (PPP1). A ênfase na elaboração do PPP é dada para o ensino contextualizado, articulando pesquisa e extensão. Neste sentido, cada documento, também descreve as características das instituições e sua inserção no Estado, os contextos demográficos e sociais que contribuíram para a construção das singularidades de cada PPP.

Os PPPs revelam os principais aspectos acerca do perfil do profissional enfermeiro que se tem formado pelas instituições em estudo. Encontra-se que o enfermeiro deve ser um profissional generalista, com competência para atuar no processo saúde-doença.
Assim, são apresentadas diferenças em cada PPP, de forma que cada um ressalta um aspecto em particular, sejam os aspectos políticos (PPP2), o tripé universitário (ensino-pesquisa-extensão) (PPP1) ou o contexto de mercado de trabalho (PPP3).

Em outro estudo(17) que também analisou os PPPs de Universidades do Ceará, viu-se que todos os PPPs das três Universidades contemplavam o perfil de egresso apresentado pelas DCNs ao referir-se ao perfil do enfermeiro com formação generalista, humanista, crítica, reflexiva.

Destaca-se que a expressão "generalista" envolve múltiplas interpretações, o que pode determinar uma indefinição sobre a intencionalidade do tipo de profissional a ser formado, como o encontrado nos diferentes PPPs. Assim, questiona-se: o perfil generalista estaria relacionado a um conhecimento geral de enfermagem em contraposição a um conhecimento especializado? O egresso de um currículo deste tipo deveria ser capacitado a atuar em todos os cenários de prática? Seu objetivo seria o de enfatizar a importância de a formação ir além dos aspectos técnicos, do saber fazer, atingindo outras competências?

Outra questão analisada refere-se aos objetivos educacionais, que em maior parte correspondem às competências e habilidades gerais dispostas nas DCN/ENF, a saber: atenção à saúde, tomada de decisão, comunicação, liderança, administração/ gerenciamento e educação permanente. Destaca-se que essas competências gerais são comuns para toda a área da Saúde e não apenas para a área de Enfermagem. Observase a expressão das competências e habilidades gerais no PPP2: "Contribuir para o desenvolvimento de competências e habilidades para realizar ações assistenciais, administrativas e educativas, visando promoção, proteção, recuperação e reabilitação da saúde do ser humano no seu ciclo vital".

As competências e habilidades contidas nas DCNs do curso de graduação de Enfermagem objetivam a integralidade da atenção, pautando essa diretriz como orientação na adequação do currículo às demandas e necessidades dos usuários do SUS(18).

Além dessas competências gerais, as DCN/ENF trazem as competências específicas e habilidades pautadas nas concepções do estudante como sujeito do seu processo formativo, da articulação entre teoria e 
prática, da diversificação dos cenários de aprendizagem, de metodologias ativas, da articulação da pesquisa com o ensino e extensão, da flexibilidade curricular, da interdisciplinaridade, da incorporação de atividades complementares, da avaliação da aprendizagem, do processo de acompanhamento, avaliação e gestão do curso, assim como a finalização do curso(19).

Com relação às competências e habilidades específicas, são apresentados nas DCN/ENF, 33 objetivos educacionais, que são contemplados de modo geral nos PPPs analisados. Esses objetivos envolvem o atual contexto de transformações da sociedade mundial, que devem considerar: as práticas educativas e de saúde expressivas da sociedade que se deseja, do profissionalcidadão que se quer formar e dos interesses do mundo do trabalho; e a requisição de habilidades e competências que tornem os profissionais aptos ao contexto das realidades, diversidades e complexidades dos cenários de prática ${ }^{(19)}$.

Os cenários de práticas e o desenvolvimento das competências gerais nos estágios supervisionados são abordados em um estudo(20) que apontou que as práticas possibilitam ao aluno a compreensão da dimensão do cuidado e o coloca como participante do processo do trabalho em saúde. De tal modo, o aluno sente a necessidade de desenvolver as competências gerais, para poder atuar nas diversas situações do contexto do trabalho em saúde.

Neste sentido, em relação à formação do egresso, a Resolução CNE/ CES n 3/ 01 recomenda que ela atenda às necessidades sociais da saúde, com ênfase no SUS, assegurando a integralidade da atenção, a qualidade e a humanização do atendimento ${ }^{(5)}$. Verificou-se que apenas o PPP3 fez referência direta ao SUS, embora o PPP1 traga à luz a intersetorialidade e a perspectiva da integralidade, o que permitiu identificar lacuna importante nesse segmento, com vistas à incorporação e divulgação do compromisso da formação profissional no âmbito do sistema político e social do país. Assim, ao discutir os processos formativos, é imprescindível identificar a concepção política e social da instituição, a lógica do serviço, se esta apresenta o seu PPP pautado nos princípios éticos e políticos da Reforma Sanitária, como destacado no PPP3: "Atender às necessidades sociais de saúde, com ênfase no Sistema Único de Saúde (SUS) e assegurando a integralidade da atenção, a qualidade $e$ humanização do atendimento".

É importante enfatizar que a mudança na graduação, a concepção ampliada de saúde, a integralidade, as práticas inovadoras, a rede de cuidados só vão efetivamente existir nos currículos e nos processos de ensino-aprendizagem, se forem objetivos a serem atingidos em todos os serviços de saúde e não somente na rede pública ${ }^{(21)}$.

A atenção integral e a responsabilidade social são mencionadas em todos os documentos analisados, sempre destacando que esse é o objeto do trabalho do enfermeiro. De fato, sabe-se que as diversas possibilidades para que se alcance e se efetive a promoção da saúde devem pautar-se nas experiências humanas nos contextos sociais, políticos, econômicos e culturais, sendo colocada em destaque a relevância dos saberes e das ações produzidas nos diferentes campos do conhecimento e das atividades a serviço da saúde ${ }^{(22)}$. É nessa perspectiva que o PPP1 enfatiza a enfermagem inserida no contexto social e intersetorial:

O PPP se apóia em um paradigma da saúde que recupera o seu significado social e traduz a necessidade do agir de forma intersetorializada numa perspectiva da integralidade. Supõe uma formação profissional que considere as estruturas políticas, institucionais, culturais da sociedade e tendências de formação e atuação para absorção dos egressos (PPP1).

O PPP2 volta-se para a necessidade do profissional estar diretamente implicado nas demandas sociais que o permeiam, reelaborando constantemente seu saber a partir destas e contribuindo para o crescimento da profissão, como destacado no trecho:

O Enfermeiro é um profissional de saúde, crítico, comprometido com as necessidades de saúde da população, com a responsabilidade de cuidar do ser humano (indivíduo, família e grupos sociais) na sua integralidade, nos níveis de atenção individual e coletiva. Deve contribuir para o desenvolvimento da profissão por meio do ensino, pesquisa, participação nas entidades de classe e no exercício da cidadania. No seu processo de formação, precisa ser preparado e estar permanentemente atualizado, não só para o desenvolvimento de uma prática competente 
voltada para resolubilidade, acessibilidade e confiabilidade do sistema de saúde brasileiro, mas também para exercer críticas às políticas nacionais de saúde (PPP2).

O PPP3 traz em seus objetivos específicos a intenção de estimular o aluno para a busca permanente do saber, desenvolvendo o aprender a aprender, aprender a fazer, aprender a viver juntos e o aprender a conhecer, na perspectiva do ensino, pesquisa e extensão. Nesse ponto, destacam-se nos PPPs, três dos quatro pilares que a UNESCO aponta como princípios fundamentais da educação para o século XXI(23):

\section{[...] Aprender a conhecer, deve priorizar os instrumentos do} conhecimento de modo a tornar-se para toda a vida 'amigo da ciência'. Aprender a fazer, propõem um trabalho que privilegie a relação teoria e prática, afim de que o aluno em formação possa por em prática os seus conhecimentos; Aprender a conviver, que representa um dos maiores desafios da educação no mundo globalizado altamente competitivo; Aprender a ser se propondo a completar o desenvolvimento do sujeito: espírito, corpo, inteligência, sensibilidade, sentido estético, responsabilidade individual e compromisso[...]" (PPP2) [...] os egressos do curso de enfermagem devem receber uma formação que esteja embasada em quatro dimensões: O saber como formação intelectual; $O$ saber ser como orientação humana profissional; O saber fazer como desempenho operativo idôneo e saber conviver, reunindo as possibilidades do conhecimento para elaboração de relações humanas edificantes e emancipadoras [...] (PPP1).

Os PPPs mostram perspectivas de formação evidenciando ainda clara referência aos processos de educação permanente que devem ser enfatizados no processo de formação profissional.

\section{As Matrizes Curriculares e sua interface com os objetivos educacionais}

O currículo acadêmico reflete uma visão de mundo, resultado de um processo de investigação determinado por um processo de pesquisa, desenvolvido no contexto de uma dada concepção de educação e de sociedade. Ele não é um conjunto isolado e estático de informações ${ }^{(24)}$.

Os conteúdos essenciais para o curso de graduação em enfermagem, conforme o estabelecido pelas
DCN/ENF devem estar relacionados com todo o processo saúde-doença do cidadão, da família e da comunidade, integrado à realidade epidemiológica e profissional, proporcionando a integralidade das ações do cuidar em enfermagem. Os conteúdos devem contemplar as ciências biológicas e da saúde, as ciências humanas e sociais e as ciências da enfermagem ${ }^{(5)}$.

Рara o presente estudo, optou-se por analisar as matrizes curriculares utilizando categorias conforme mecionado anteriormente na metodologia. Desta forma: conteúdos biológicos/individuais quando as disciplinas fizerem referência ao modelo biomédico, ou seja, as disciplinas que contemplam todo o conteúdo base dos cursos da área da saúde. Quando nos referimos às disciplinas que trazem relação com atividades coletivas e preventivas, estamos fazendo referência aos conteúdos que se voltam para o que, durante muito tempo nos currículos, convencionou-se chamar de saúde pública, ou seja, aquelas disciplinas que têm uma vertente maior na prevenção dos agravos.

Já quando o foco são as disciplinas voltadas para a prevenção e atenção individual/ biológica nos referimos àquelas com cunho curativista, estando a prevenção nesse quesito ligada às condutas hospitalocêntricas e focadas na acurácia da técnica. O último quesito diz respeito às disciplinas que se voltam para as ciências humanas e sociais, como observa-se a seguir no Quadro 1. 
Quadro 1: Demonstrativo das matrizes curriculares quanto à distribuição da carga horária dos quesitos em análise em relação á carga horária total do curso. Fortaleza, CE, 2011.

\begin{tabular}{|c|c|c|c|}
\hline Matrizes Curriculares & PPP1 & PPP2 & PPP3 \\
\hline \multirow{2}{*}{ Disciplinas voltadas para conteúdos biológicos e individuais } & 1008h/a & 1184h/a & $1152 \mathrm{~h} / \mathrm{a}$ \\
\hline & $(25,60 \%)$ & $(29,95 \%)$ & $(27,35 \%)$ \\
\hline \multirow{2}{*}{ Disciplinas relacionadas a ações coletivas e preventivas } & $624 \mathrm{~h} / \mathrm{a}$ & $832 \mathrm{~h} / \mathrm{a}$ & $900 \mathrm{~h} / \mathrm{a}$ \\
\hline & $(15,85 \%)$ & $(21,05 \%)$ & $(21,36 \%)$ \\
\hline \multirow{2}{*}{ Disciplinas que abrangem a prevenção e atenção individual/ biológica } & $1824 \mathrm{~h} / \mathrm{a}$ & $1584 \mathrm{~h} / \mathrm{a}$ & 1728h/a \\
\hline & $(46,35 \%)$ & $(40,10 \%)$ & $(41,04 \%)$ \\
\hline \multirow{2}{*}{ Disciplinas das ciências sociais e humanas } & $480 \mathrm{~h} / \mathrm{a}$ & $352 \mathrm{~h} / \mathrm{a}$ & $432 \mathrm{~h} / \mathrm{a}$ \\
\hline & $(12,20 \%)$ & $(8,90 \%)$ & $(10,25 \%)$ \\
\hline
\end{tabular}

A análise das matrizes curriculares mostrou que as disciplinas relacionadas aos conteúdos biológicos/individuais perfazem um percentual de 25,6\%, $29,95 \%$ e $27,35 \%$, respectivamente nos PPPs 1,2 e 3 . Já as disciplinas que trazem relação com atividades coletivas e preventivas, os percentuais são 15,85\%, 21,05\% e $21,36 \%$. E as que tratam da atenção sob a vertente da prevenção e da atenção individual/biológica, o percentual ficou em $46,35 \%, 40,1 \%$ e $41,04 \%$. Já às das ciências sociais e humanas foi $12,2 \%, 8,9 \%$ e $10,25 \%$.

Identifica-se que as três instituições concentram sua maior carga horária no tópico que versa sobre a prevenção e a atenção individual/biológica. Nesse ponto, enfatiza-se novamente que foram incluídas neste quesito, disciplinas mais voltadas para o contexto de atenção hospitalar. Assim, estão contempladas disciplinas como as que tratam da parte de Semiologia e Semiotécnica.

Uma dificuldade a ser destacada nesse ponto, diz respeito aos termos excessivamente genéricos adotados para denominar algumas disciplinas, o que não possibilita ter a certeza de seu conteúdo teórico. Essa dificuldade é evidente em um dos PPPs que não apresenta as ementas das disciplinas/módulos no documento, deixando dúvidas quanto ao conteúdo abordado.

O predomínio, nas matrizes curriculares, de disciplinas pautadas nas ciências biológicas reflete o modelo biomédico. O modelo que vê o corpo humano como uma máquina muito complexa, com partes que se inter-relacionam, obedecendo a leis natural e psicologicamente perfeitas, pressupõe, ainda, que a máquina complexa (o corpo) precisa constantemente de inspeção. Acredita-se que as doenças são resultado ou de processo degenerativo dentro do corpo, ou de agentes químicos, físicos ou biológicos que o invadem, ou, ainda, da falha de algum mecanismo regulatório do organismo. Segundo essa visão, doenças podem ser detectadas apenas por métodos científicos ${ }^{(24)}$. O sujeito implicado é desvalorizado na sua capacidade de opinar e participar da produção da saúde e na busca de estratégias para melhorar a sua qualidade de vida(13). Questão esta que vem sendo evidenciada na falta de adesão aos tratamentos das doenças crônicas.

Nesta concepção mecanicista, o processo de trabalho é centrado no serviço e profissionais, dando origem a modelos de organização curricular baseados em relações de causa e efeito, o que se contrapõe ao princípio da integralidade preconizado pelo SUS.

É preciso desenvolver no profissional, desde a sua formação, o conhecimento para a compreensão complexa da realidade e de valores presentes nas questões humanas, científicas e sociais. O desafio é ampliar a visão dos alunos para além das perspectivas biológicas e médico-centradas no processo saúde-doença(13). Deve haver relação integrativa entre os conteúdos presentes nos currículos e as necessidades do processo de ensinoaprendizagem.

\section{CONSIDERAÇÕES FINAIS}

A análise permitiu verificar que os Projetos Políticos Pedagógicos e Matrizes Curriculares dos cursos de graduação de Enfermagem analisados, se encontram estruturados segundo as Diretrizes Curriculares Nacionais, com ênfase na formação do enfermeiro generalista, mas com competência para atuar no processo saúde-doença de forma contextualizada e articulada com os movimentos sociais e políticos da sociedade.

No entanto, apreendeu-se a ênfase em segmentos diferentes na formação do enfermeiro por parte de cada 
curso, pautada na formação integrada ao ensino-pesquisa e extensão versus para o mercado de trabalho versus enquanto sujeito social, o que evidencia aspectos filosóficos distintos por parte dos cursos, merecedores de debates e discussão na integração formativa profissional.

No que tange às competências e habilidades gerais, os PPPs convergem na importância para a educação permanente como perspectiva na formação do enfermeiro da atualidade. Sabe-se que a Política Nacional de Educação Permanente em Saúde (PNEPS) foi implantada como uma das estratégias do SUS para mudar a questão da formação profissional em saúde, o que se configura como estimulante, a ciência das necessidades de mudança para o SUS.

Em controvérsia à política do SUS, os PPPs dos cursos de graduação em Enfermagem do presente estudo, apresentam matrizes curriculares com concentração de maior carga horária na prevenção e na atenção individual/biológica, e disciplinas voltadas para o foco de atenção ao contexto hospitalar, o que denota ainda fragilidade рага a mudança na formação do enfermeiro, pautado no modelo da doença para a integralidade.

\section{REFERÊNCIAS}

1. Merhy EE. Saúde: a cartografia do trabalho vivo. São Paulo: Hucitec; 2002.

2. Assis MMA, Cerqueira EM, Nascimento MAA, Santos AM, Jesus WLAL. Atenção Primária à Saúde e sua articulação com a Estratégia Saúde da Família: construção política metodológica e prática. Revista de APS. 2007;10:189-199.

3. Brasil. Constituição (1988). Constituição da República Federativa do Brasil: promulgada em 5 de outubro de 1988. Brasília, DF: Senado, 1988.

4. Conselho Nacional de Educação, Câmara de Educação Superior, Ministério da Educação. Parecer CNE/CES n. 1133, de 7 agosto de 2001 - Institui as diretrizes curriculares nacionais dos cursos de graduação em enfermagem, medicina e nutrição. Brasília (Brasil): Ministério da Educação, 2001.

5. Brasil, Ministério da Educação. Resolução CNE/CES Nº 03 de $7 / 11 / 2001$ - Institui as diretrizes curriculares nacionais do curso de graduação em Enfermagem. Brasília: 2001.

6. Saupe R, Cestari ME. O trabalho coletivo na construção do projeto político pedagógico dos cursos de enfermagem. Rev. Eletr. Enf. [Internet]. 2002 [cited 2011 nov 14];4(2):22 -26. Available from:

http://www.fen.ufg.br/fen_revista/revista4_2/pdf/coletivo.pdf 7. Veiga IPA. Projeto político-pedagógico: continuidade ou transgressão para acertar? In: Castanho S, Castanho MELM, editors. O que há de novo na educação superior: do projeto pedagógico à prática transformadora. São Paulo: Papirus; 2000:183-217.

8. Silva EVM, Oliveira MS, Silva SF, Lima VV. A formação de profissionais de saúde em sintonia com o SUS: currículo integrado e interdisciplinar. Brasília (Brasil): CONASEMS; 2008.
Pontua-se aqui, a necessidade do desenvolvimento de estratégias para discussão e debates acerca da formação do enfermeiro, nos diversos segmentos da formação profissional, seja nas universidades, serviços e espaços sociais pertinentes à socialização reflexiva e estratégica para a formação do enfermeiro no contexto do SUS.

A produção do cuidado do enfermeiro permeada pelos dispositivos: acolhimento, escuta, autonomia, coresponsabilização do usuário e família, permite uma relação de pertença e vínculo com o serviço de saúde, estratégias que podem ser incorporadas nas tecnologias do cuidado de enfermagem, como o processo de enfermagem, desde que desenvolvido de forma contextualizada à realidade de aplicação. Evidencia-se então, a necessidade da integração ensino-serviço com a inserção da academia como agente de mudança e transformação da prática de enfermagem.

Acredita-se que pesquisas como esta, contribuem para revisão dos PPPs das universidades e debates acerca da temática na pesquisa, ensino e extensão, sendo importante veículo de repercussão no âmbito da formação profissional.

9. Ciuffo RS, Ribeiro VMB. Sistema Único de Saúde e a formação dos médicos: um diálogo possível? Interface - Comunic., Saúde, Educ. 2008;12(14):11-20.

10. Fernandes JD, Xavier IM, Ceribelli MIPF, Bianco MHC, Maeda $D$, Rodrigues MVC. Diretrizes curriculares e estratégias para implantação de uma nova proposta pedagógica. Rev Esc Enferm USP. 2005;39(4):443-9.

11. Ceccim RB, Feuerwerker LCM. Mudança na graduação dos profissionais de saúde sob o eixo da Integralidade. Cad Saude Publica. 2004;20:1400-1410.

12. Prado ML, Backes VMS, Santana ME, Souza ML. Políticas Publicas na formação em saúde: contribuição da enfermagem para superação das desigualdades regionais brasileiras. Texto Contexto Enferm. 2007;16(3):531-5.

13. Brasil. Ministério da Saúde. Núcleo Técnico da Política de Humanização. Humaniza SUS: a clínica ampliada. Brasília: Ministério da Saúde; 2004.

14.Virgilio MSTG, Oliveira NR. Considerações sobre a clínica ampliada no processo de enfermagem. Saúde Coletiva. 2010; 07(38):61-66.

15. Silva RPG, Rodrigues RM. Sistema Único de Saúde e a graduação em enfermagem no Paraná. Rev Bras Enferm. 2010;63(1): 66-72.

16. Pimentel, A. O método da análise documental: seu uso numa pesquisa historiográfica. Cad Pesqui. 2001;114:179-95.

17. Silva MJ, Sousa EM, Freitas CL. Formação em enfermagem: interface entre as diretrizes curriculares e os conteúdos de atenção básica. Rev Bras Enferm.2011;64(2):315-21.

18. Santana FR, Nakatani AYK, Souza ACS, Casagrande LDR, Esperidião, E. Diretrizes Curriculares Nacionais do Curso de 
Graduação em Enfermagem: Uma Visão Dialética. Revista Eletrônica de Enfermagem. 2005;7(3):294-300.

19. Lopes ND, Teixeira E, Vale EG, Cunha FS, Xavier IM, Fernandes JD et al. Aderência dos Cursos de Graduação em Enfermagem às Diretrizes Curriculares Nacionais. Rev. bras. enferm. 2007;60(6):627-634.

20. Benito GAV, Tristão KM, Paula ACSF, Santos MA, Ataide LJ, Lima RCD. Desenvolvimento de competências gerais durante o estágio supervisionado. Rev. bras. enferm. 2012;65(1):172-178. 21. Feuerwerker LM. Estratégias atuais para a mudança na graduação das profissões da saúde. Cadernos ABEM. 2006;2. 22. Chrizostimo MM, Rosas AMMTF. A trilogia da promoção em saúde, consulta de enfermagem e gestão em saúde: o entrelaçar reflexivo. Informe-se em promoção da saúde. 2006;2(2):09-10.

23. Delors J. Educação: um tesouro a descobrir. Relatório para a UNESCO da Comissão Internacional sobre Educação para o século XXI. São Paulo: Cortez Editora; 1999.

24. Koifman, L. O modelo biomédico e a reformulação do currículo médico da Universidade Federal Fluminense. Hist Cienc Saude Manguinhos. 2001;8(1):48-70.

Artigo recebido em 08/12/2011.

Aprovado para publicação em 06/08/2012.

Artigo publicado em 31/03/2013. 